\title{
Uniqueness of the Kadomtsev-Petviashvili and Boussinesq Equations
}

\author{
Wen-Xiu Ma ${ }^{\mathrm{a}}$ and Aslı Pekcan ${ }^{\mathrm{b}}$ \\ a Department of Mathematics and Statistics, University of South Florida, Tampa, FL 33620- \\ 5700, USA \\ b Department of Mathematics, Istanbul University, 34134, Vezneciler, Istanbul, Turkey \\ Reprint requests to W.-X. M.; Tel.: (813)974-9563, Fax: (813)974-2700, E-mail: mawx@ @as.usf.edu
}

Z. Naturforsch. 66a, 377 -382 (2011); received December 2, 2010

The Kadomtsev-Petviashvili and Boussinesq equations $\left(u_{x x x}-6 u u_{x}\right)_{x}-u_{t x} \pm u_{y y}=0,\left(u_{x x x}-\right.$ $\left.6 u u_{x}\right)_{x}+u_{x x} \pm u_{t t}=0$, are completely integrable, and in particular, they possess the three-soliton solution. This article aims to expose a uniqueness property of the Kadomtsev-Petviashvili (KP) and Boussinesq equations in the integrability theory. It is shown that the Kadomtsev-Petviashvili and Boussinesq equations and their dimensional reductions are the only integrable equations among a class of generalized Kadomtsev-Petviashvili and Boussinesq equations $\left(u_{x_{1} x_{1} x_{1}}-6 u u_{x_{1}}\right)_{x_{1}}+$ $\sum_{i, j=1}^{M} a_{i j} u_{x_{i} x_{j}}=0$, where the $a_{i j}$ 's are arbitrary constants and $M$ is an arbitrary natural number, if the existence of the three-soliton solution is required.

Key words: Integrable Equations; Hirota's Bilinear Form; Three-Soliton Condition. PACS numbers: 02.30.Ik; 02.30.Xx; 05.45.Yv

\section{Introduction}

It is interesting to search for nonlinear integrable equations and study their integrable characteristics in mathematical physics. The task is remarkably difficult due to the nonlinearity involved. No general theory is available for dealing with nonlinear differential equations, indeed. Each method focuses on a specific aspect or is based on a specific mathematical subject.

Hirota's bilinear method [1], however, proposes a direct algebraic approach to nonlinear integrable equations [1-3], and it is pretty powerful in presenting multi-soliton solutions, particularly three-soliton solutions $[2,4]$. It is a common sense that the existence of the three-soliton solution usually implies the integrability [5] of the considered equations.

In this article, we will consider a class of generalized Kadomtsev-Petviashvili (KP) and Boussinesq equations:

$$
\left(u_{x_{1} x_{1} x_{1}}-6 u u_{x_{1}}\right)_{x_{1}}+\sum_{i, j=1}^{M} a_{i j} u_{x_{i} x_{j}}=0,
$$

where $M$ is a natural number and we assume that the constants $a_{i j}$ 's satisfy the symmetric property $a_{i j}=a_{j i}$, $1 \leq i, j \leq M$, without loss of generality. This is the most general class of generalizations of the stationary Korteweg-de Vries (KdV) equation by adding the second-order partial derivatives. Using the Hirota bilinear technique, we would like to show a kind of uniqueness property for the KP and Boussinesq equations

$$
\begin{aligned}
& \left(u_{x x x}-6 u u_{x}\right)_{x}-u_{t x} \pm u_{y y}=0 \\
& \left(u_{x x x}-6 u u_{x}\right)_{x}+u_{x x} \pm u_{t t}=0
\end{aligned}
$$

in mathematical physics. That is, we will show that among the above class of nonlinear differential equations, the KP and Boussinesq equations and their dimensional reductions are the only integrable equations, if the existence of the three-soliton solution is required. We also mention that Hirota's bilinear method is used to determine nonlinear superposition formulas for the $\mathrm{KP}$ and Boussinesq equations [6, 7].

\section{The Three-Soliton Condition}

A general Hirota bilinear equation reads

$$
P\left(\mathrm{D}_{x}, \mathrm{D}_{t}, \cdots\right) f \cdot f=0,
$$

where $P$ is a polynomial in the indicated variables just to satisfy

$$
P(0,0, \cdots)=0
$$


and $\mathrm{D}_{x}, \mathrm{D}_{t}, \cdots$ are Hirota's differential operators defined by

$$
\begin{aligned}
\mathrm{D}_{y}^{p} f(y) \cdot g(y) & =\left.\left(\partial_{y}-\partial_{y^{\prime}}\right)^{p} f(y) g\left(y^{\prime}\right)\right|_{y^{\prime}=y} \\
& =\left.\partial_{y^{\prime}}^{p} f\left(y+y^{\prime}\right) g\left(y-y^{\prime}\right)\right|_{y^{\prime}=0}, p \geq 1 .
\end{aligned}
$$

Let us introduce new variables

$$
\eta_{m}=k_{m} x+\omega_{m} t+\cdots+\eta_{m, 0}, m \geq 1,
$$

and define a set of constants

$$
A_{m n}=-\frac{P\left(\bar{p}_{m}-\bar{p}_{n}\right)}{P\left(\bar{p}_{m}+\bar{p}_{n}\right)}, m, n \geq 1,
$$

where the involved parameters

$$
\bar{p}_{m}=\left(k_{m}, \omega_{m}, \cdots\right), m \geq 1,
$$

satisfy the dispersion relations

$$
P\left(\bar{p}_{m}\right)=0, m \geq 1,
$$

and $\eta_{m, 0}, m \geq 1$, are arbitrary constant shifts. Obviously, we have the one-soliton and two-soliton solutions to the bilinear equation (3):

$$
\begin{aligned}
f= & 1+\varepsilon \mathrm{e}^{\eta_{1}}, f=1+\varepsilon\left(\mathrm{e}^{\eta_{1}}+\mathrm{e}^{\eta_{2}}\right) \\
& +\varepsilon^{2} A_{12} \mathrm{e}^{\eta_{1}+\eta_{2}},
\end{aligned}
$$

where $\varepsilon$ is an arbitrary perturbation parameter. Noting that

$$
P\left(\mathrm{D}_{x}, \mathrm{D}_{t}, \cdots\right) \mathrm{e}^{\eta_{1}} \cdot \mathrm{e}^{\eta_{2}}=P\left(\bar{p}_{1}-\bar{p}_{2}\right) \mathrm{e}^{\eta_{1}+\eta_{2}},
$$

the existence of the two-soliton solution requires that the constant $A_{12}$ must be determined by (6). The oneperiodic and two-periodic wave solutions have the same situation of existence of solutions [8].

However, in general, we do not have the threesoliton solution automatically. Let us fix

$$
\begin{aligned}
f= & 1+\varepsilon\left(\mathrm{e}^{\eta_{1}}+\mathrm{e}^{\eta_{2}}+\mathrm{e}^{\eta_{3}}\right)+\varepsilon^{2}\left(A_{12} \mathrm{e}^{\eta_{1}+\eta_{2}}\right. \\
& \left.+A_{13} \mathrm{e}^{\eta_{1}+\eta_{3}}+A_{23} \mathrm{e}^{\eta_{2}+\eta_{3}}\right)+\varepsilon^{3} A_{123} \mathrm{e}^{\eta_{1}+\eta_{2}+\eta_{3}},
\end{aligned}
$$

where $A_{123}=A_{12} A_{13} A_{23}$ and $\varepsilon$ is an arbitrary perturbation parameter. Then generally we have a three-soliton condition

$$
\begin{gathered}
\sum_{\sigma_{1}, \sigma_{2}, \sigma_{3}= \pm 1} P\left(\sigma_{1} \bar{p}_{1}+\sigma_{2} \bar{p}_{2}+\sigma_{3} \bar{p}_{3}\right) P\left(\sigma_{1} \bar{p}_{1}-\sigma_{2} \bar{p}_{2}\right) \\
\cdot P\left(\sigma_{2} \bar{p}_{2}-\sigma_{3} \bar{p}_{3}\right) P\left(\sigma_{1} \bar{p}_{1}-\sigma_{3} \bar{p}_{3}\right)=0,
\end{gathered}
$$

to guarantee the existence of the three-soliton solution (10). If this condition is automatically satisfied, then the considered equation (3) is called integrable in the sense of existence of the three-soliton solution.

Let us now turn back to the class of nonlinear equations defined by (1). It is direct to see that under the dependent variable transformation

$$
u=-2(\ln f)_{x_{1} x_{1}}
$$

every nonlinear equation defined by (1) can be written as

$$
\begin{gathered}
P\left(\mathrm{D}_{x_{1}}, \mathrm{D}_{x_{2}}, \cdots, \mathrm{D}_{x_{M}}\right) f \cdot f= \\
\left(\mathrm{D}_{x_{1}}^{4}+\sum_{i, j=1}^{M} a_{i j} \mathrm{D}_{x_{i}} \mathrm{D}_{x_{j}}\right) f \cdot f=0,
\end{gathered}
$$

which exactly gives

$$
\begin{aligned}
& f_{x_{1} x_{1} x_{1} x_{1}} f-4 f_{x_{1} x_{1} x_{1}} f_{x_{1}}+3 f_{x_{1} x_{1}}^{2} \\
& +\sum_{i, j=1}^{M} a_{i j}\left(f f_{x_{i} x_{j}}-f_{x_{i}} f_{x_{j}}\right)=0 .
\end{aligned}
$$

We assume that the three-soliton solution $f$ to (13) is given by (10) with

$$
\begin{aligned}
& \eta_{m}=k_{m} x_{1}+\sum_{j=2}^{M} l_{m, j} x_{j}+\eta_{m, 0}, \\
& A_{m n}=-\frac{R_{m n}}{S_{m n}}, 1 \leq m, n \leq 3,
\end{aligned}
$$

where

$$
\begin{aligned}
R_{m n}= & \left(k_{m}-k_{n}\right)^{4}+a_{11}\left(k_{m}-k_{n}\right)^{2} \\
& +\sum_{j=2}^{M} 2 a_{1 j}\left(k_{m}-k_{n}\right)\left(l_{m, j}-l_{n, j}\right) \\
& +\sum_{i, j=2}^{M} a_{i j}\left(l_{m, i}-l_{n, i}\right)\left(l_{m, j}-l_{n, j}\right), 1 \leq m, n \leq 3, \\
S_{m n}= & \left(k_{m}+k_{n}\right)^{4}+a_{11}\left(k_{m}+k_{n}\right)^{2} \\
& +\sum_{j=2}^{M} 2 a_{1 j}\left(k_{m}+k_{n}\right)\left(l_{m, j}+l_{n, j}\right) \\
& +\sum_{i, j=2}^{M} a_{i j}\left(l_{m, i}+l_{n, i}\right)\left(l_{m, j}+l_{n, j}\right), 1 \leq m, n \leq 3 .
\end{aligned}
$$

Taking advantage of the dispersion relations of (1), $P\left(\bar{p}_{m}\right)=0, \bar{p}_{m}=\left(k_{m}, l_{m, 2}, \cdots, l_{m, M}\right), 1 \leq m \leq 3$,

which leads to

$k_{m}^{4}=-a_{11} k_{m}^{2}-\sum_{j=2}^{M} 2 a_{1 j} k_{m} l_{m, j}-\sum_{i, j=2}^{M} a_{i j} l_{m, i} l_{m, j}$,

$1 \leq m \leq 3$

we can expand the three-soliton condition (11) for (1) and show that the three-soliton condition (11) is equiv- 
alent to a determinant relation

$k_{1}^{2} k_{2}^{2} k_{3}^{2} \sum_{i, j, p, q=2}^{M} a_{i j} a_{p q} \operatorname{det}\left(K, L_{i}, L_{p}\right) \operatorname{det}\left(K, L_{j}, L_{q}\right)$

$=0$,

where

$K=\left(k_{1}, k_{2}, k_{3}\right)^{\mathrm{T}}, L_{i}=\left(l_{1, i}, l_{2, i}, l_{3, i}\right)^{\mathrm{T}}, 2 \leq i \leq M$.

The proof is given in the appendix. Obviously, as an example, the three-soliton condition (17) gives rise to

$$
k_{1}^{2} k_{2}^{2} k_{3}^{2}\left(a_{22} a_{33}-a_{23}^{2}\right) \operatorname{det}\left(K, L_{2}, L_{3}\right)^{2}=0,
$$

when $M=3$ [9].

The condition (17) is an integrability condition for the bilinear equation (13). Not every equation in (1) has this property, and two counterexamples are the $(2+1)$ dimensional Boussinesq equation [10]

$$
\left(u_{x x x}-6 u u_{x}\right)_{x}+u_{x x}-u_{t t}+u_{y y}=0,
$$

and the $(3+1)$-dimensional KP equation [11]

$$
\left(u_{x x x}-6 u u_{x}\right)_{x}-u_{t x}+u_{y y}+u_{z z}=0 .
$$

\section{Uniqueness Property}

Based on the above three-soliton condition (17), we would like to prove that for whatever value $M$, any nonlinear equation defined by (1) can be transformed into one of the KP and Boussinesq equations (2) and their dimensional reductions. This exposes a uniqueness property of the KP and Boussinesq equations in the integrability theory. The result includes all cases of the value of $M$, generalizing the case $M \leq 3$ discussed in [9].

In what follows, let us present our proof in five steps.

Step 1: Take an invertible linear transform of independent variables

$$
\begin{aligned}
& X_{2}=Q Y_{2}, X_{2}=\left(x_{2}, \cdots, x_{M}\right)^{\mathrm{T}}, \\
& Y_{2}=\left(y_{2}, \cdots, y_{M}\right)^{\mathrm{T}},
\end{aligned}
$$

where $Q$ is an orthogonal matrix transforming the symmetric matrix

$$
A_{2}=\left(a_{i j}\right)_{2 \leq i, j \leq M}
$$

into a diagonal matrix:

$$
Q^{\mathrm{T}} A_{2} Q=\operatorname{diag}\left(b_{2}, \cdots, b_{M}\right) .
$$

Therefore, under the transform (22), we have

$$
\sum_{i, j=2}^{M} a_{i j} u_{x_{i} x_{j}}=\sum_{j=2}^{M} b_{j} u_{y_{j} y_{j}}
$$

and further, an original equation defined by (1) becomes

$$
\begin{aligned}
& \left(u_{x_{1} x_{1} x_{1}}-6 u u_{x_{1}}\right)_{x_{1}}+a_{11} u_{x_{1} x_{1}}+\sum_{j=2}^{M} c_{1 j} u_{x_{1} y_{j}} \\
& +\sum_{j=2}^{M} b_{j} u_{y_{j} y_{j}}=0
\end{aligned}
$$

for some constants $c_{1 j}, 2 \leq j \leq M$.

Step 2: Now, apply the three-soliton condition (17) to the transformed equation (26), and then we see from the arbitrariness character of the parameters $l_{i, j}$ that there is at most one non-zero constant, let us say $b_{2}$, among the coefficients $b_{i}, 2 \leq i \leq M$.

Step 3: Assume that there is at least one non-zero constant, say $c_{13} \neq 0$, among the coefficients $c_{1 j}, 3 \leq$ $j \leq M$. Then making another invertible linear transform of independent variables

$$
\begin{aligned}
& r=x_{1}, s=y_{2}, \\
& \left(t, z_{4}, \cdots, z_{M}\right)^{\mathrm{T}}=R\left(y_{3}, y_{4}, \cdots, y_{M}\right)^{\mathrm{T}},
\end{aligned}
$$

where the invertible constant matrix $R$ satisfies

$$
R\left(c_{13}, c_{14}, \cdots, c_{1 M}\right)^{\mathrm{T}}=\left(c_{13}, 0 \cdots, 0\right)^{\mathrm{T}},
$$

the transformed equation (26) becomes

$$
\begin{aligned}
& \left(u_{r r r}-6 u u_{r}\right)_{r}+a_{11} u_{r r}+c_{12} u_{r s} \\
& +c_{13} u_{r t}+b_{2} u_{s s}=0 .
\end{aligned}
$$

This equation with $c_{13}=0$ corresponds to the transformed equation (26) with all $c_{1 j}=0,3 \leq j \leq M$. Therefore, we only need to consider (29) with arbitrary constant coefficients.

Step 4: Let $b_{2}=0$. If $c_{12}=c_{13}=0$, then (29) becomes the stationary Boussinesq equation when $a_{11} \neq 0$ and the stationary derivative $\mathrm{KdV}$ equation 
when $a_{11}=0$, both of which are the dimensional reductions of the KP and Boussinesq equations. Otherwise, let us assume $c_{12} \neq 0$ without loss of generality, and choose two constants $\alpha$ and $\beta$ satisfying

$$
a_{11}+\alpha c_{12}+\beta c_{13}=0
$$

Then the invertible linear transform of $r, s$, and $t$,

$$
r^{\prime}=r+\alpha s+\beta t, t^{\prime}=c_{12} t-c_{13} s, s^{\prime}=s,
$$

can transform (29) into

$$
\left(u_{r^{\prime} r^{\prime} r^{\prime}}-6 u u_{r^{\prime}}\right)_{r^{\prime}}+c_{12} u_{r^{\prime} s^{\prime}}=0 .
$$

This presents the derivative $\mathrm{KdV}$ equation - the dimensional reduction of the KP equation.

Step 5: Let $b_{2} \neq 0$. Then an invertible linear transform of independent variables,

$$
r^{\prime}=r-\frac{c_{12}}{2 b_{2}} s, t^{\prime}=t, s^{\prime}=s,
$$

removes the mixed partial-derivative term $u_{r s}$, and (29) becomes

$$
\begin{aligned}
& \left(u_{r^{\prime} r^{\prime} r^{\prime}}-6 u u_{r^{\prime}}\right)_{r^{\prime}}+\left(a_{11}-\frac{c_{12}^{2}}{4 b_{2}}\right) u_{r^{\prime} r^{\prime}} \\
& +c_{13} u_{r^{\prime} t^{\prime}}+b_{2} u_{s^{\prime} s^{\prime}}=0 .
\end{aligned}
$$

Now if $c_{13}=0$, then this presents the Boussinesq equation, and it can be further transformed into the standard Boussinesq equation for whatever values of $a_{11}, c_{12}$, and $b_{2} \neq 0$ [12]. If $c_{13} \neq 0$, then under a further invertible linear transform of independent variables

$r^{\prime \prime}=r^{\prime}-\left(\frac{a_{11}}{c_{13}}-\frac{c_{12}^{2}}{4 c_{13} b_{2}}\right) t^{\prime}, t^{\prime \prime}=t^{\prime}, s^{\prime \prime}=s^{\prime}$,

(34) becomes

$\left(u_{r^{\prime \prime} r^{\prime \prime} r^{\prime \prime}}-6 u u_{r^{\prime \prime}}\right)_{r^{\prime \prime}}+c_{13} u_{r^{\prime \prime} t^{\prime \prime}}+b_{2} u_{s^{\prime \prime} s^{\prime \prime}}=0$,

which presents the KP equation.

\section{Concluding Remarks}

To conclude, we discussed a class of generalized KP and Boussinesq equations (1), and proved that among the considered class of equations, the only integrable equations are the KP and Boussinesq equations (2) and their dimensional reductions. This shows that the KP and Boussinesq equations possess a uniqueness prop- erty in the integrability theory, presenting a kind of particular integrable equations. In particular, the $(2+1)$ dimensional Boussinesq equation (20) and the $(3+1)$ dimensional KP equation (21) do not have the threesoliton solution (see also [13, 14] for exact solutions to the $(3+1)$-dimensional KP equation).

In analyzing the existence of the three soliton solution for the generalized KP and Boussinesq equations (1), the difficulty is to compute the three-soliton condition (11), and our success is to rewrite the three-soliton condition (11) as a determinant relation (17), which is put in the appendix. An approach of Darboux transformations [15] could be used to generate multi-soliton solutions directly from the three-soliton solution.

There are various discussions about the $(2+1)$-dimensional Boussinesq equation and the $(3+1)$-dimensional KP equation as well as another class of higherdimensional generalizations of the Boussinesq equation [16]. Those equations are shown to be connected with Ricatti-type integrable ordinary differential equations, and correspondingly, abundant exact solutions can be worked out [16-20].

\section{Acknowledgements}

The work was supported in part by the Established Researcher Grant, the CAS faculty development grant, and the CAS Dean research grant of the University of South Florida, Chunhui Plan of the Ministry of Education of China, the State Administration of Foreign Experts Affairs of China, and the Scientific and Technological Research Council of Turkey.

\section{Appendix: A Proof of the Three-Soliton Determinant Condition}

We verify that the three-soliton condition (11) can be written as a determinant relation (17). Noting the even property of the polynomial

$$
P\left(x_{1}, x_{2}, \cdots, x_{M}\right)=x_{1}^{4}+\sum_{i, j=1}^{M} a_{i j} x_{i} x_{j}
$$

for the generalized KP and Boussinesq bilinear equation (13), the three-soliton condition (11) can be computed as follows:

$$
\begin{aligned}
& \text { Sum }:=\frac{1}{2} \sum_{\sigma_{1}, \sigma_{2}, \sigma_{3}= \pm 1} P\left(\sigma_{1} \bar{p}_{1}+\sigma_{2} \bar{p}_{2}+\sigma_{3} \bar{p}_{3}\right) \\
& \cdot P\left(\sigma_{1} \bar{p}_{1}-\sigma_{2} \bar{p}_{2}\right) P\left(\sigma_{2} \bar{p}_{2}-\sigma_{3} \bar{p}_{3}\right) P\left(\sigma_{1} \bar{p}_{1}-\sigma_{3} \bar{p}_{3}\right)
\end{aligned}
$$


$=\sum_{\left(\sigma_{1}, \sigma_{2}, \sigma_{3}\right) \in S} P\left(\sigma_{1} \bar{p}_{1}+\sigma_{2} \bar{p}_{2}+\sigma_{3} \bar{p}_{3}\right)$

$\cdot P\left(\sigma_{1} \bar{p}_{1}-\sigma_{2} \bar{p}_{2}\right) P\left(\sigma_{2} \bar{p}_{2}-\sigma_{3} \bar{p}_{3}\right) P\left(\sigma_{1} \bar{p}_{1}-\sigma_{3} \bar{p}_{3}\right)$, where $S=\{(1,1,1),(1,1,-1),(1,-1,1),(-1,1,1)\}$ and $\bar{p}_{m}, 1 \leq m \leq 3$, are defined as in (15). Then we expand it to obtain

$$
\begin{aligned}
& \text { Sum }=576\left(k_{1}^{6} k_{3}^{6} k_{2}^{4}+k_{2}^{6} k_{3}^{6} k_{1}^{4}+k_{1}^{6} k_{2}^{6} k_{3}^{4}\right)+1152 a_{11}\left(k_{1}^{6} k_{2}^{4} k_{3}^{4}+k_{1}^{4} k_{2}^{6} k_{3}^{4}+k_{1}^{4} k_{2}^{4} k_{3}^{6}\right)+1728 a_{11}^{2} k_{1}^{4} k_{2}^{4} k_{3}^{4} \\
& +1152 \sum_{j=2}^{M} a_{1 j}\left(k_{1}^{3} k_{2}^{6} k_{3}^{4} l_{1, j}+k_{1}^{6} k_{2}^{3} k_{3}^{4} l_{2, j}+k_{1}^{4} k_{2}^{3} k_{3}^{6} l_{2, j}+k_{1}^{4} k_{2}^{6} k_{3}^{3} l_{3, j}+k_{1}^{6} k_{2}^{4} k_{3}^{3} l_{3, j}+k_{1}^{3} k_{2}^{4} k_{3}^{6} l_{1, j}\right) \\
& +2304 \sum_{i, j=2}^{M} a_{1 i} a_{1 j}\left(k_{1}^{4} k_{2}^{3} k_{3}^{4} l_{2, i} l_{3, j}+k_{3}^{4} k_{1}^{3} k_{2}^{3} l_{2, j} l_{1, i}+k_{2}^{4} k_{1}^{3} k_{3}^{3} l_{1, j} l_{3, i}\right) \\
& +2304 a_{11} \sum_{j=2}^{M} a_{1 j}\left(k_{1}^{4} k_{2}^{3} k_{3}^{4} l_{2, j}+k_{1}^{4} k_{2}^{4} k_{3}^{3} l_{3, j}+k_{1}^{3} k_{2}^{4} k_{3}^{4} l_{1, j}\right) \\
& +1152 a_{11} \sum_{i, j=2}^{M} a_{i j}\left(k_{1}^{3} k_{2}^{3} k_{3}^{4} l_{1, i} l_{2, j}+k_{1}^{4} k_{2}^{3} k_{3}^{3} l_{2, i} l_{3, j}+k_{1}^{3} k_{2}^{4} k_{3}^{3} l_{1, j} l_{3, i}\right) \\
& +2304 k_{1}^{3} k_{2}^{3} k_{3}^{3} \sum_{i, p, q=2}^{M} a_{1 i} a_{p q}\left(l_{1, i} l_{2, p} l_{3, q}+l_{1, p} l_{2, q} l_{3, i}+l_{1, q} l_{2, i} l_{3, p}\right) \\
& +1152 \sum_{i, j=2}^{M} a_{i j}\left(k_{1}^{3} k_{2}^{3} k_{3}^{6} l_{1, i} l_{2, j}+k_{1}^{6} k_{2}^{3} k_{3}^{3} l_{2, i} l_{3, j}+k_{1}^{3} k_{2}^{6} k_{3}^{3} l_{1, j} l_{3, i}\right) \\
& +576 \sum_{i, j, p, q=2}^{M} a_{i j} a_{p q}\left(2 k_{1}^{3} k_{3}^{2} k_{2}^{3} l_{1, i} l_{3, j} l_{2, p} l_{3, q}+2 k_{1}^{3} k_{3}^{3} k_{2}^{2} l_{1, i} l_{2, j} l_{2, p} l_{3, q}+2 k_{1}^{2} k_{3}^{3} k_{2}^{3} l_{1, i} l_{2, j} l_{1, p} l_{3, q}\right. \\
& \left.-k_{1}^{4} k_{3}^{2} k_{2}^{2} l_{2, i} l_{3, j} l_{2, p} l_{3, q}-k_{2}^{4} k_{3}^{2} k_{1}^{2} l_{1, i} l_{3, j} l_{1, p} l_{3, q}-k_{2}^{2} k_{3}^{4} k_{1}^{2} l_{1, i} l_{2, j} l_{1, p} l_{2, q}\right) .
\end{aligned}
$$

Plugging a consequence of the dispersion relations (16),

$$
k_{m}^{6}=-a_{11} k_{m}^{4}-\sum_{j=2}^{M} 2 a_{1 j} k_{m}^{3} l_{m, j}-\sum_{i, j=2}^{M} a_{i j} k_{m}^{2} l_{m, i} l_{m, j}, 1 \leq m \leq 3,
$$

into the above expression and carrying out cancelations, we have

$$
\begin{aligned}
& \text { Sum }=576 k_{1}^{2} k_{2}^{2} k_{3}^{2}\left[k_{2}^{2}\left(\sum_{i, j=2}^{M} a_{i j} l_{1, i} l_{1, j}\right)\left(\sum_{i, j=2}^{M} a_{i j} l_{3, i} l_{3, j}\right)+k_{1}^{2}\left(\sum_{i, j=2}^{M} a_{i j} l_{2, i} l_{2, j}\right)\left(\sum_{i, j=2}^{M} a_{i j} l_{3, i} l_{3, j}\right)\right. \\
& \left.+k_{3}^{2}\left(\sum_{i, j=2}^{M} a_{i j} l_{1, i} l_{1, j}\right)\left(\sum_{i, j=2}^{M} a_{i j} l_{2, i} l_{2, j}\right)\right]-1152 k_{1}^{2} k_{2}^{2} k_{3}^{2}\left[k_{1} k_{2}\left(\sum_{i, j=2}^{M} a_{i j} l_{1, i} l_{2, j}\right)\left(\sum_{i, j=2}^{M} a_{i j} l_{3, i} l_{3, j}\right)\right. \\
& \left.+k_{1} k_{3}\left(\sum_{i, j=2}^{M} a_{i j} l_{3, i} l_{1, j}\right)\left(\sum_{i, j=2}^{M} a_{i j} l_{2, i} l_{2, j}\right)+k_{2} k_{3}\left(\sum_{i, j=2}^{M} a_{i j} l_{2, i} l_{3, j}\right)\left(\sum_{i, j=2}^{M} a_{i j} l_{1, i} l_{1, j}\right)\right] \\
& +576 k_{1}^{2} k_{2}^{2} k_{3}^{2}\left(2 k_{1} k_{2} \sum_{i, j, p, q=2}^{M} a_{i j} a_{p q} l_{1, i} l_{3, j} l_{2, p} l_{3, q}+2 k_{1} k_{3} \sum_{i, j, p, q=2}^{M} a_{i j} a_{p q} l_{1, p} l_{2, j} l_{2, q} l_{3, i}\right. \\
& +2 k_{2} k_{3} \sum_{i, j, p, q=2}^{M} a_{i j} a_{p q} l_{1, q} l_{2, i} l_{1, j} l_{3, p}-k_{1}^{2} \sum_{i, j, p, q=2}^{M} a_{i j} a_{p q} l_{2, i} l_{3, j} l_{2, p} l_{3, q}
\end{aligned}
$$


$\left.-k_{2}^{2} \sum_{i, j, p, q=2}^{M} a_{i j} a_{p q} l_{1, j} l_{3, i} l_{1, q} l_{3, p}-k_{3}^{2} \sum_{i, j, p, q=2}^{M} a_{i j} a_{p q} l_{1, i} l_{2, j} l_{1, p} l_{2, q}\right)$

$=576 k_{1}^{2} k_{2}^{2} k_{3}^{2} \sum_{i, j, p, q=2}^{M} a_{i j} a_{p q} \operatorname{det}\left(K, L_{i}, L_{p}\right) \operatorname{det}\left(K, L_{j}, L_{q}\right)$.

This implies that the three-soliton determinant condition (17) holds for the generalized KP and Boussinesq bilinear equation (13).

[1] R. Hirota, The Direct Method in Soliton Theory, Cambridge University Press, Cambridge 2004.

[2] J. Hietarinta, J. Math. Phys. 28, 1732 (1987).

[3] W.X. Ma and W. Strampp, Phys. Lett. A 341, 441 (2005).

[4] J. Hietarinta, J. Math. Phys. 28, 2094 (1987).

[5] W. X. Ma, in: Encyclopedia of Nonlinear Science (Ed. A. Scott), Taylor \& Francis, New York, 2005 pp. 450453.

[6] A. Nakamura, J. Math. Phys. 22, 2456 (1981).

[7] X. B. Hu, Y. Li, and Q. M. Liu, Acta Math. Appl. Sin.E. 9, 17 (1993).

[8] W. X. Ma, R. G. Zhou, and L. Gao, Mod. Phys. Lett. A 21, 1677 (2009).

[9] A. Pekcan, J. Nonlin. Math. Phys. 16, 127 (2009).

[10] R. S. Johnson, J. Fluid Mech. 323, 65 (1996).

[11] E. A. Kuznetsov and S. K. Turitsyn, Soviet Phys. JETP 82, 1457 (1982).
[12] W. X. Ma, C. X. Li, and J. S. He, Nonlin. Anal.-Theor. 70, 4245 (2009).

[13] W. X. Ma, Commun. Nonlin. Sci. 16, 2663 (2011).

[14] W. X. Ma and E. G. Fan, Comp. Math. Appl. 61, 950 (2011).

[15] T. Aktosun and C. van der Mee, Inverse Probl. 25, 105003 (2009).

[16] Z. Y. Yan, Phys. Lett. A 361, 223 (2007).

[17] M. A. Allen and G. Rowlands, Phys. Lett. A 235, 145 (1997).

[18] Y. Chen, Z. Y. Yan, and H. Q. Zhang, Phys. Lett. A 307, 107 (2003).

[19] S. M. El-Sayed and D. Kaya, Appl. Math. Comput. 157, 523 (2004)

[20] A. S. A. Rady, E. S. Osmana, and M. Khalfallah, Appl. Math. Comput. doi:10.1016/j.amc.2009.05.028. 